\title{
Induction of apoptosis in glioma cells requires cell-to-cell contact with human umbilical cord blood stem cells
}

\author{
CHRISTOPHER S. GONDI ${ }^{1}$, VENKATESWARA R. GOGINENI ${ }^{1}$, CHANDRAMU CHETTY $^{1}$, VENKATA R. DASARI ${ }^{1}$, \\ BHARATHI GORANTLA ${ }^{1}$, MEENA GUJRATI ${ }^{2}$, DZUNG H. DINH ${ }^{3}$ and JASTI S. RAO ${ }^{1,3}$ \\ Departments of ${ }^{1}$ Cancer Biology and Pharmacology, ${ }^{2}$ Pathology, and ${ }^{3}$ Neurosurgery, \\ University of Illinois College of Medicine at Peoria, Peoria, IL 61605, USA
}

Received January 7, 2010; Accepted February 23, 2010

DOI: 10.3892/ijo_00000599

\begin{abstract}
We have previously demonstrated the multipotent nature of human umbilical cord blood stem cells (hUCB). In this study, we have attempted to show the use of hUCB in glioma therapy. We used hUCB enriched in CD44 and CD133 cells for our studies and observed that glioma cells co-cultured with hUCB undergo apoptosis. To prove the role of cell-tocell contact in the induction of apoptotic events, we used a modified $0.22 \mu \mathrm{m}$ Boyden's chamber where the upper surface was used to culture glioma cells (SNB19 or U87) or xenografts (4910 or 5310) and the lower surface to culture hUCB. TUNEL assay was carried out to determine the degree of apoptotic induction and we observed that glioma or xenograft cells cocultured with hUCB had a higher number of TUNEL-positive characteristics $(63 \pm 6 \%)$ compared to the controls. Further, we co-cultured glioma cells labeled with lipophilic green fluorescent dye and hUCB labeled with lipophilic red fluorescent dye. FACS analysis of cells collected from the upper and lower surfaces revealed that glioma cells had taken up red fluorescent dye from the stem cells $(70 \pm 3 \%)$ when compared to glioma cells co-cultured with fibroblast cells $(15 \pm 4 \%)$. The apoptotic events in the glioma and xenograft cells co-cultured with hUCB were also confirmed by Western blot analysis for the cleavage of PARP and activation of caspase 8 . In addition, elevated levels of CHK-2 levels and downregulation of MAP2K1 were observed in glioma cells co-cultured with hUCB indicating the DNA damage and decrease in cell survival. Nude mice, intracranially implanted with luciferase-expressing U87 cells followed by implantation of hUCB or human fibroblast cells showed retardation of intracranial tumors in hUCB-implanted mice. Taken together, these results demonstrate that hUCB have therapeutic potential with possible clinical implications.
\end{abstract}

Correspondence to: Dr Jasti S. Rao, Department of Cancer Biology and Pharmacology, University of Illinois College of Medicine at Peoria, One Illini Drive, Peoria, IL 61605, USA

E-mail: jsrao@uic.edu

Key words: glioma, stem cells, apoptosis, CD133, CD44

\section{Introduction}

Stem cells are promising tools on which the field of regenerative medicine relies for the treatment of human pathologies. Stem cells can be obtained from various sources, including embryos, fetal tissues, umbilical cord blood, and also terminally differentiated organs. Once forced to expand and differentiate into functional progenies, stem cells may become suitable for cell replacement and tissue engineering. The manipulation and/or stimulation of adult stem cells seems to be particularly promising, as it could improve the endogenous regenerative potential without risks of rejection and overcome the ethical and political issues related to embryonic stem cell research. Despite an incomplete knowledge of the genetic control program driving their fate and plasticity, stem cells are already leaving the bench and reaching the bedside. Glial tumors invariably recur due to the regrowth of invasive cells, which are unaffected by standard treatment modalities. Drivers of glioma invasion include autocrine signals propagated by secreted factors that signal through receptors on the tumor. These secreted factors are able to diffuse through the peritumoral stroma, thereby influencing parenchymal cells that surround the tumor mass.

Mesenchymal stem cells (MSC), which reside within the stromal compartment of the bone marrow, have the capacity to differentiate into cells of connective tissue lineages $(1,2)$ and cells that are not a part of their normal repertoire (3-5). Recent studies have indicated that MSC have the capacity to target therapeutic genes for malignant glioma $(6,7)$. Thus, therapies involving genes such as interleukin (IL) 2 (7) and interferon- $\beta$ (8), which employ MSCs as a targeting vehicle, may potentially allow for the development of a novel therapeutic approach for malignant glioma. As the notion of unorthodox plasticity in MSC remains somewhat interesting $(9,10)$, the use of rat MSC as direct immune effector cells, rather than simple gene vehicles, presents results regarding the direct cytotoxic effect of rat MSC against rat malignant glioma cells (11).

Human umbilical cord blood (hUCB) is a rich source of both hematopoietic stem cells (12) and MSC (13). In addition, hUCB-derived stem cells evidence higher proliferation and expansion potential than has been observed in their adult bone marrow counterparts (14). Furthermore, the authors have detected more cytotoxic capability of immune effector cells from hUCB than with cells derived from peripheral blood 
against malignant glioma cells and medulloblastoma cells (15). Therefore, hUCB may be considered to be an alternative source of MSC (13).

Recently, researchers have demonstrated the cytotoxicity of human umbilical cord blood-derived mesenchymal stem cells against human malignant glioma cells. In this study, the authors used hUCB-derived MSC, which were activated by interleukin-2, interleukin-15, granulocyte macrophage colonystimulating factor, and others. The authors demonstrated that the hUCB-derived MSC activated with cytokines showed significantly higher cytotoxicity than that observed with unactivated hUCB-derived MSC (16). As demonstrated, the authors used the co-culture method to obtain the effector/ target $(\mathrm{E} / \mathrm{T})$ cell ratios of $0: 1,1: 1,5: 1$, and 10:1 (16). A recent report has demonstrated that rat umbilical cord blood stem cells completely abolish rat mammary carcinomas with no evidence of metastasis or recurrence at 100 days post-tumor cell inoculation (17). In this study, as a necessary preliminary step, they evaluated the effect of unengineered rat umbilical cord blood stem cells on Matt B III cancer cells in vitro and in vivo. To their surprise, the stem cells them-selves mediated a profound anti-tumor effect both in vivo and in vitro (17). In the present study, we demonstrate this anti-tumor effect in vivo and in vitro using human glioma cell lines and xenografts. We used hUSC positive for CD133 and CD44 expression and we have attempted to demonstrate that the induction of cytotoxicity by hUSC on human glioma cells requires cell-to-cell contact.

\section{Materials and methods}

Isolation of CD133 and CD44 positive hUCB cells. After obtaining informed consent, human umbilical cord blood was collected from healthy volunteers according to a protocol approved by the Institutional Review Board. After the process of birth, but prior to the release of the placenta, the umbilical cord was drained of blood by inserting an intravenous needle into the umbilical cord and allowing the blood to flow by gravity into collection bags containing anti-coagulant. Soon after collection, the cord blood was centrifuged over ficol as per standard protocol and the buffy coat collected. Mesenchymal stem cells were separated by selectively precipitating non-mesenchymal cells with appropriate antibodies using the Rosette mesenchymal separating kit. To determine the presence of mesenchymal stem cells in the cellular isolate, the cells were cultured in knockout media supplemented with $10 \%$ knockout serum replacement in humidified $5 \% \mathrm{CO}_{2}$ atmosphere. To isolate CD133 and CD44 positive cells, the collected cells were cultured on $100 \mathrm{~mm}$ plates to $40 \%$ confluence after which the cells were scraped and incubated with antiCD133, FITC-conjugated antibody followed by anti-CD44, $\mathrm{Tx}$ red-conjugated antibody in serum-free media for $30 \mathrm{~min}$ at $37^{\circ} \mathrm{C}$ in a $5 \% \mathrm{CO}_{2}$ atmosphere. The cells were then sorted by FACS analysis to isolate both CD44 and CD133 positive cells in a Becton-Dickinson flow cytometer. The collected cells were then cultured either in methyl cellulose-containing media to determine embryoid body formation (18) or in knockout media as previously described.

Cell lines and culture conditions. Glioma cell lines SNB19 and U87 and human glioma xenograft 4910 and 5310 cells were maintained as monolayers in DMEM/F12 medium supplemented with $10 \% \mathrm{FBS}, 50 \mathrm{U} / \mathrm{ml}$ penicillin and $50 \mu \mathrm{g} / \mathrm{ml}$ streptomycin (Life Technologies Inc., Frederick, MD) at $37^{\circ} \mathrm{C}$ in a humidified $5 \% \mathrm{CO}_{2}$ atmosphere.

Modified Boyden's chamber model for the growth of hUCB stem cells with glioma cells. To determine the interaction of hUCB stem cells with glioma cells, we used the modified Boyden's chamber model also termed as semi-co-cultures. In this method, we used a transwell chamber with a $0.22 \mu \mathrm{m}$ pore size to keep the hUCB stem cells and glioma cells separate. Both the dorsal and ventral surfaces of the transwell membrane were coated with Matrigel to facilitate cell adhesion. hUCB stem cells were grown on the dorsal surface and glioma cells were grown on the ventral surface. Cells were cultured in the transwell chamber in knockout media supplemented with $10 \%$ knockout serum replacement in humidified $5 \% \mathrm{CO}_{2}$ atmosphere.

In situ terminal-deoxy-transferase-mediated dUTP nick end labeling (TUNEL) assay. A TUNEL apoptosis detection kit (Upstate Biotechnology Inc., Lake Placid, NY) was used for DNA fragmentation fluorescence staining as per the manufacturer's protocol. Briefly, $72 \mathrm{~h}$ after glioma cells (SNB19, U87, 4910 and 5310) were grown on the lower surface of the transwell chamber with or without hUCB cells on the upper chamber, the hUCB layer was scraped off. The glioma cell layer was fixed with $4 \%$ paraformaldehyde $0.1 \mathrm{M}$ phosphate buffer ( $\mathrm{pH}$ 7.4). Cells were incubated with a reaction mix containing biotin-dUTP and terminal deoxynucleotidyl transferase for $60 \mathrm{~min}$. Fluorescein-conjugated avidin was applied to the sample, which was then incubated in the dark for $30 \mathrm{~min}$. Positively stained, fluorescein-labeled cells were visualized by fluorescence microscopy and quantified.

Identification of hUCB glioma cell contact in the Boyden's chamber model. To determine the interaction of hUCB cells with glioma cells, we used the modified Boyden's chamber model. In this method, we used a transwell chamber with a $0.22 \mu \mathrm{m}$ pore size to keep the hUCB stem cells and glioma cells separate as previously described. In this experimental set up, the hUCB stem cells were labeled with the lipophilic cell tracker red dye. The interaction of the hUCB stem cell membrane with the glioma cell membrane should cause the transfer of the lipophilic dye to the glioma cell layer. Briefly, glioma cells (SNB19, U87, 4910 and 5310) were grown on the lower surface of the transwell chamber with or without hUCB cells on the upper chamber. Seventy-two hours after culture, the hUCB layer was scraped off and the glioma cell layer was counterstained with a nuclear stain (DAPI) to visualize the nucleus and photographed. These cells were then scraped out and sorted by FACS analysis to validate the visual observation of cell tracker red stain transfer to glioma cells from hUCB cells. Seven experimental sets were set up to determine the change in hUCB-glioma cell contact, if any, over time. Periodically $(0,6,12,20,24,30$ and $72 \mathrm{~h})$, the upper hUCB cell layers were scraped and the lower glioma layer stained with DAPI and observed for red fluorescence.

Western blot analysis. hUCB stem cells were cultured with human glioma cells in the modified Boyden's chamber model 
A

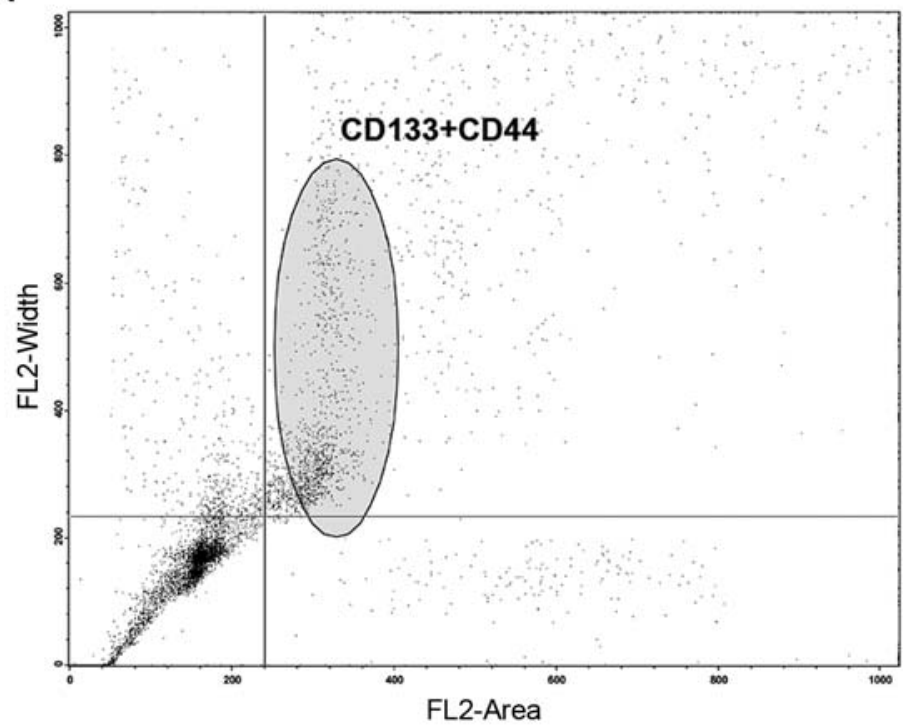

B

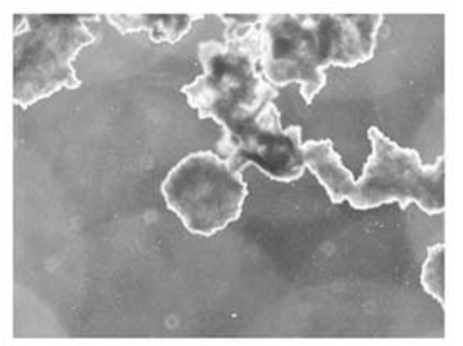

C

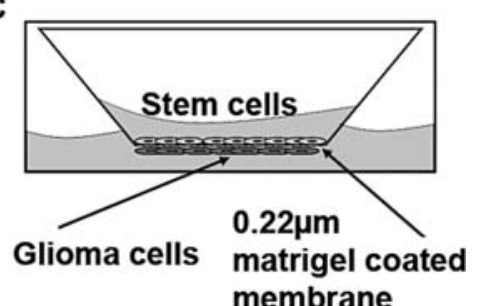

Figure 1. Human umbilical cord blood cells were isolated by standard Ficol gradient separation. To separate stem cells (hUCB) from the nucleated cell population of the umbilical cord blood, cells were labeled with anti CD44-FITC and anti CD44-Tx Red antibody and separated by flow cytometry (A). To determine the ability of the isolated cells to form embryoid bodies, cells were cultured in media containing methyl cellulose (B). To determine the interaction of hUSC with glioma cells, a modified Boyden's chamber was used. The chamber consisted of a porous membrane ( $0.22 \mu \mathrm{m}$ pore size), which was coated with Matrigel to facilitate the growth of cells on both the upper and lower surfaces. Human umbilical cord blood stem cells were grown on the upper surface and glioma cells (SNB19, U87, 5310 or 4910) were grown on the lower surface (C).

as previously described with glioma cells on the lower surface and hUCB stem cells on the upper surface. The glioma cell layers were scraped after $72 \mathrm{~h}$ of culture and cells were lysed with a Nonidet P-40 (NP-40) buffer containing 0.3\% NP-40, $142 \mathrm{mM} \mathrm{KCl}, 5 \mathrm{mM} \mathrm{MgCl}, 2 \mathrm{mM}$ ethylenediaminetetraacetic acid (EDTA), $20 \mathrm{mM}$ HEPES (pH 7.4), and a cocktail of protease inhibitors (aprotinin, leupeptin and phenylmethylsulfonyl fluoride) (Sigma, St. Louis, MO). Total protein (10-50 $\mu \mathrm{g}$ ) was separated on a $12 \%$ SDS-PAGE (sodium dodecyl sulfate-polyacrylamide gel electrophoresis) and subsequently transferred to a polyvinylidene difluoride membrane (Bio-Rad, Hercules, CA). The membranes were blocked with $6 \%$ non-fat dry milk and probed with antihuman caspase 8 or anti-human PARP antibody. Appropriate secondary antibody conjugated with horseradish peroxidase was used and membranes were developed using enhanced chemiluminescence protocol as per the manufacturer's instructions (Amersham, Arlington Heights, IL). To determine the induction of apoptosis, the levels of cleaved caspase 8 and PARP were assessed and quantified.

Reverse transcription PCR. Total RNA was isolated from control and glioma cells grown in contact with hUCB stem cells from the Boyden's chamber model as previously described using TRIzol according to standard protocol after $72 \mathrm{~h}$ of culture. Total RNA was treated with DNase I (Invitrogen, Carlsbad, CA) to remove contaminating genomic DNA. First strand cDNA was prepared using Superscript III reverse transcriptase (Invitrogen). One hundred nanograms of first strand cDNA were used for PCR amplification using standard protocols. We used the following primers for MAP2K1: F-5'-GCGTCTAAGTGTTTGGGAAG-3' and R5'-CCAAGCACAAAGCCAATCCA-3'. For CHK-2, we used the following primers: F-5'-TTTTGAACATTTCTCCA
TTTTCC-3' and R-5'-CATCAGTGACTGTGAAAAAG CAA-3'. Quantitative analysis was done using real-time PCR analysis.

Nude mice model to assess glioma cytotoxicity by hUCB cells. To determine in vivo the interactions of hUCB stem cells with glioma cells, nude mice were implanted intracranially with luciferase-expressing U87 cells. Seven days after implantation, the mice were implanted with hUCB stem cells. Tumor progression was determined by periodic imaging with the Xenogen imaging system. Four weeks after implantation, mice were sacrificed and brains harvested. Brains were fixed in formaldehyde followed by paraffin embedding and sectioning as per standard protocols. Paraffin sections were stained with H\&E to determine tumor location and progression. In vivo tumor size is represented as photon counts per second and graphically shown.

\section{Results}

Isolation of CD133 and CD44 positive cells from human umbilical cord blood. Human umbilical cord blood (hUCB) has been proven to be a valuable source of hematopoietic stem cells, but its therapeutic potential is known to extend beyond the hematopoietic component and evidence suggests regenerative potential in solid organs as well. There is evidence that other stem or progenitor populations, such as MSC, exist in hUCB, which might be responsible for these effects. Many different stem and progenitor cell populations have been isolated with potential ranging from embryonic-like to lineagecommitted progenitor cells. In this experiment, we used a flow cytometer sorter programmed to isolate cells positive for both CD44 and CD133. From the FACS scatter plot, we observed that the CD44 and CD133 population of cells is very 


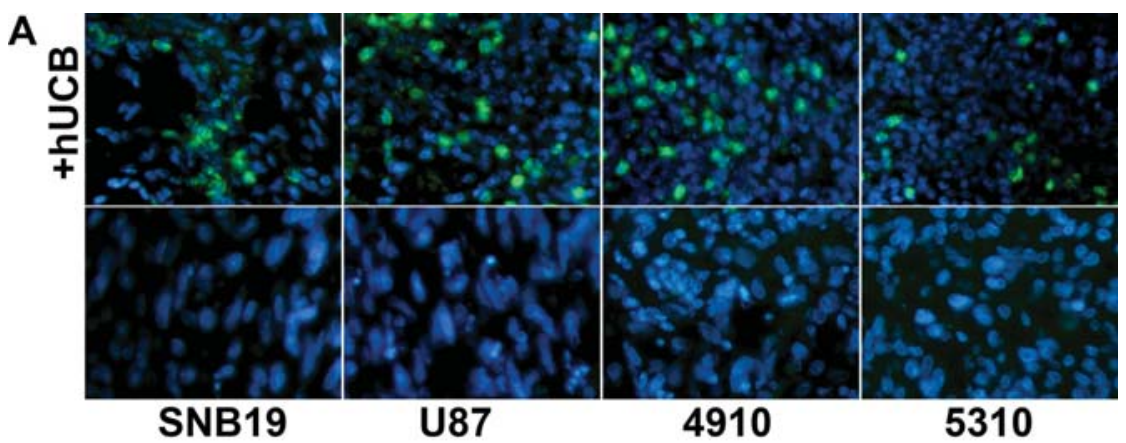

B

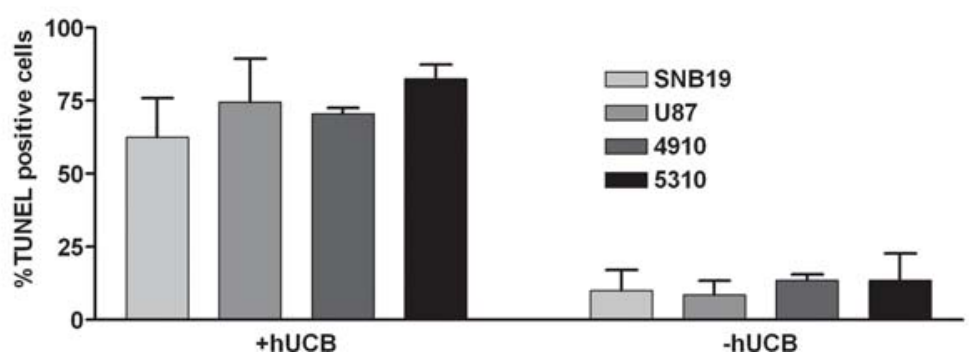

Figure 2. To determine the interaction of hUCB with glioma cells, a modified Boyden's chamber was used. The chamber consisted of a porous membrane $(0.22 \mu \mathrm{m}$ pore size $)$, which was coated with Matrigel to facilitate the growth of cells on both the upper and lower surfaces. Human umbilical cord blood stem cells were grown on the upper surface and glioma cells (SNB19, U87, 5310 or 4910) were grown on the lower surface as previously described. TUNEL assay was performed on the lower glioma surface after scraping off the upper hUCB layer (A). Quantitative analysis for the number of TUNEL-positive cells was carried out and graphically represented as a percentage of TUNEL-positive cells (B).

small (Fig. 1A). Expansion of these cells on methyl cellulosecontaining media (18) showed embryoid body formation (Fig. 1B), which is indicative of stem cell character. These cells were then cultured on the Boyden's chamber (Fig. 1C) with or without glioma cells on the lower surface.

hUCB stem cells cause apoptotic induction in glioma cells (SNB19, U87, 4910 and 5310). To determine the interaction of hUCB stem cells with glioma cells, we used a modified Boyden's chamber model. The chamber consisted of a porous membrane $(0.22 \mu \mathrm{m}$ pore size $)$, which was coated with Matrigel to facilitate the growth of cells on both the upper and lower surfaces. Human umbilical cord blood stem cells were grown on the upper surface and glioma cells (SNB19, U87, 5310 or 4910) were grown on the lower surface as previously described. TUNEL assay was performed on the lower glioma surface after scraping off the upper hUCB stem cell layer. From the TUNEL assay, we observed that glioma cells grown in the presence of hUCB stem cells resulted in TUNEL-positive cells as observed by green fluorescence (Fig. 2A). No significant change in apoptotic events were observed between the different glioma cells lines (data not shown). Quantitative analysis revealed that glioma cells cultured in the presence of hUCB cells had increased apoptosis by up to $75 \%$ (Fig. 2B). In contrast, no significant increase in apoptosis was observed when glioma cells were cultured alone.

Cell-to-cell interaction between glioma cells and hUCB stem cells involves transfer of hUCB membrane components to glioma cells. Many of the membrane processes involve perturbations that affect the structural or dynamic properties of the phospholipid bilayer or result in changes to the cell membrane or its morphology. Numerous membrane studies employing model systems have been reported and varied techniques have been developed for imaging surface phenomena in living cells. Here, we used hUCB stem cells labeled with lipophilic dye cell tracker red. To determine cell-to-cell interaction, glioma cells were separated from hUCB stem cells via the $0.22 \mu \mathrm{m}$ membrane in the Boyden's chamber model as described in Materials and methods. In all of the conditions where glioma cells were cultured with hUCB cells, we observed that the cell tracker red dye was transferred to the glioma cells (Fig. 3A). From the time course studies, we observed that maximum dye transfer $(58 \pm 6 \%)$ occurred $30 \mathrm{~h}$ after culture and no significant increase was observed until $72 \mathrm{~h}(68 \pm 3 \%)$ in glioma cells (Fig. 3B). FACS analysis confirmed an increase in the number of glioma cells with red dye (Fig. 3C).

Contact between hUCB stem cells and glioma cells activates caspase 8 and PARP cleavage. To determine the effect of the interaction of hUCB stem cells with glioma cells, we used the modified Boyden's chamber model. Briefly, the chamber consisted of a porous membrane $(0.22 \mu \mathrm{m}$ pore size $)$, which was coated with Matrigel to facilitate the growth of cells on both the upper and lower surfaces. Human umbilical cord blood stem cells were grown on the upper surface and glioma cells (SNB19, U87, 5310 or 4910) were grown on the lower surface as previously described. The glioma cells were scraped and Western blot analysis was carried out as per standard protocol for caspase 8 and PARP. The results demonstrated that glioma cells, which were grown in contact with hUCB cells, exhibited cleavage of caspase 8 and PARP (Fig. 4A). As would be expected given the levels of cleaved caspase 8 and PARP, quantitative analysis revealed that up to $50 \%$ of the cells were apoptotic (Fig. 4B). 


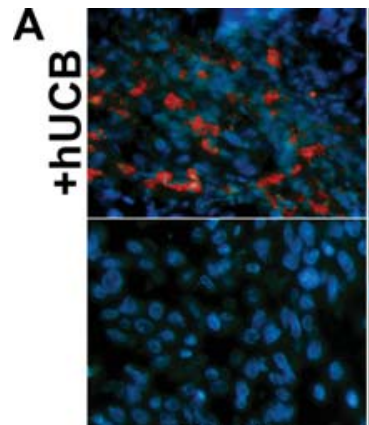

SNB19

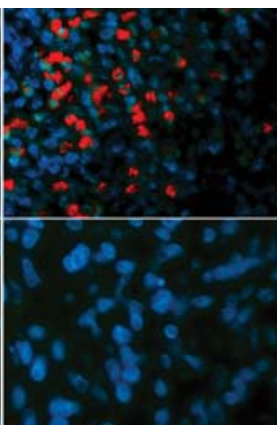

U87

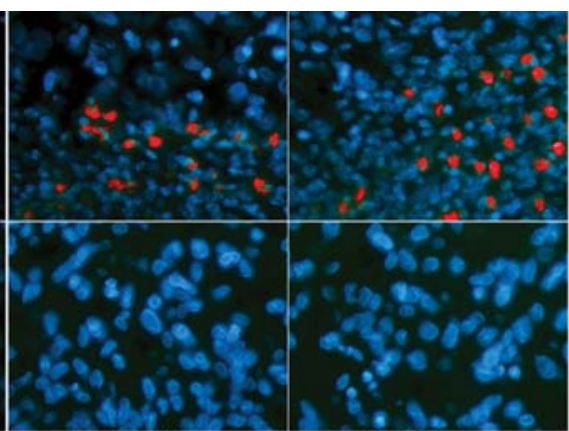

4910
5310

B

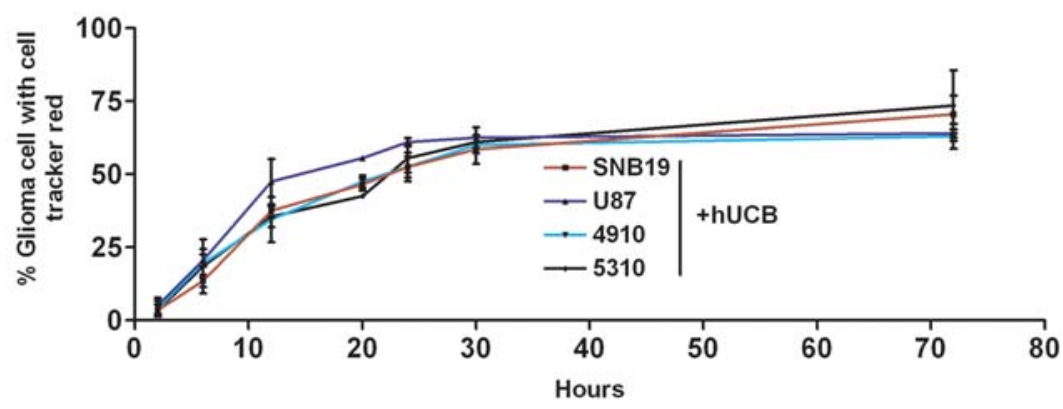

\section{C}
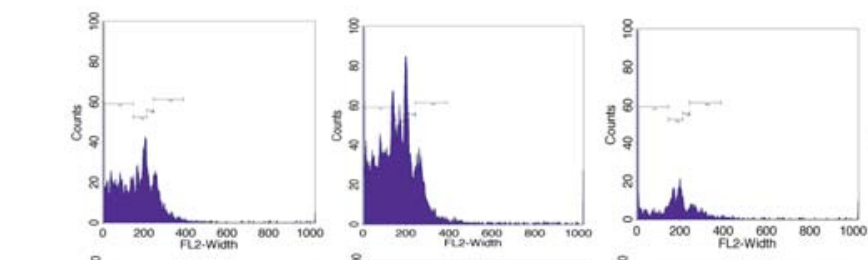

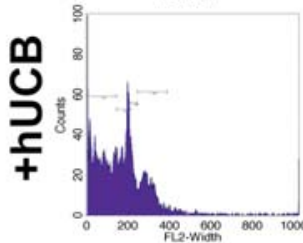

SNB19

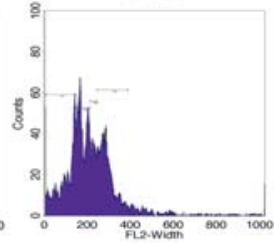

U87

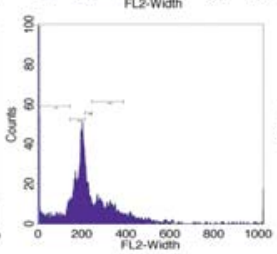

4910
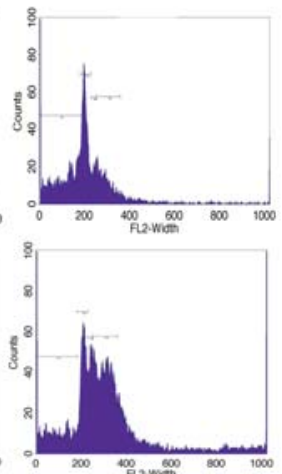

5310

Figure 3. To determine the interaction of hUCB with glioma cells, a modified Boyden's chamber was used. The chamber consisted of a porous membrane $(0.22 \mu \mathrm{m}$ pore size $)$, which was coated with Matrigel to facilitate the growth of cells on both the upper and lower surfaces. Human umbilical cord blood stem cells were grown on the upper surface and glioma cells (SNB19, U87, 5310 or 4910) were grown on the lower surface as previously described. The experiment was performed in multiple sets to determine cell-to-cell interaction over time. The cells were allowed to grow for $72 \mathrm{~h}$ under standard cell culture conditions. Periodic images were taken at different time intervals $(6,12,20,24,36$ and $72 \mathrm{~h})$ of the lower glioma cell surface after the hUCB layer was scraped off (A) and the percentage of glioma cells with cell tracker red stain were determined and plotted over time (B). After 72 h, glioma cells were trypsinized from the lower surface of the membrane and sorted for cell tracker red fluorescence by FACS analysis (C).

Contact of hUCB cells with glioma cells upregulates $\mathrm{CHK}-2$ and downregulates MAP $2 K 1$. To determine the effect of the interaction of hUCB stem cells with glioma cells, we used the modified Boyden's chamber model. Briefly, the chamber consisted of a porous membrane $(0.22 \mu \mathrm{m}$ pore size $)$, which was coated with Matrigel to facilitate the growth of cells on both the upper and lower surfaces. Human umbilical cord blood stem cells were grown on the upper surface and glioma cells (SNB19, U87, 5310 or 4910) were grown on the lower surface as previously described. From the Western blot analysis, we observed that in all of the glioma cells cultured in contact with hUCB stem cells, levels of CHK-2 increased, which is indicative of cell cycle arrest. Furthermore, levels of MAP2K1 decreased, which is indicative of cellular changes in the Ras signaling pathway (Fig. 5A). Quantitative analysis revealed that CHK-2 levels increased more than 10 -fold in glioma cells that were cultured in contact with hUCB stem cells whereas no significant change was observed when glioma cells were cultured alone. Similarly, MAP2K1 levels were downregulated more than 15 -fold in glioma cells that were cultured in contact with hUCB stem cells whereas no significant change was observed when glioma cells were cultured alone (Fig. 5B).

hUCB stem cells inhibit establishment of intracranial tumors in nude mice. To determine the anti-tumor effect of hUCB stem cells under in vivo conditions, we implanted nude mice with $1 \times 10^{6}$ luciferase-expressing U87 cells. Seven days after implantation, mice were implanted again with $1 \times 10^{5} \mathrm{hUCB}$ stem cells or $10 \mu \mathrm{l}$ of PBS at the site of U87 implantation. Tumor growth was monitored by periodic imaging with the Xenogen imaging system after intraperitoneal injections of 
A

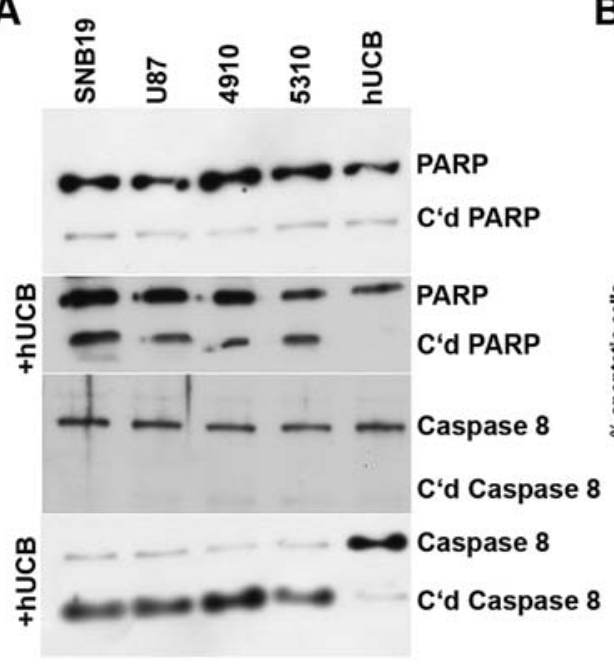

B

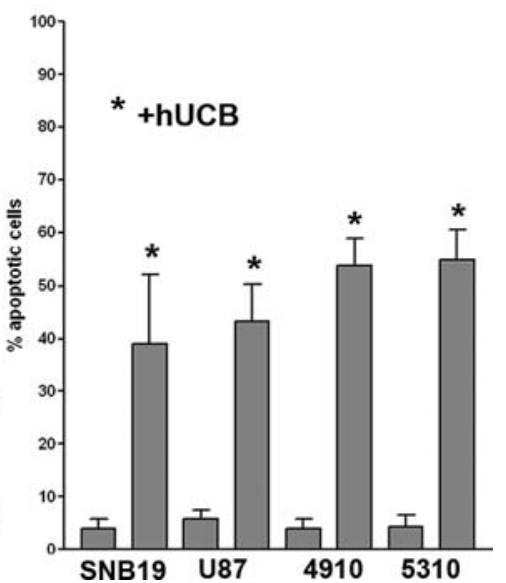

Figure 4. To determine the interaction of hUCB with glioma cells, a modified Boyden's chamber was used. The chamber consisted of a porous membrane $(0.22 \mu \mathrm{m}$ pore size), which was coated with Matrigel to facilitate the growth of cells on both the upper and lower surfaces. After $72 \mathrm{~h}$ of culture, the lower glioma cell surface was scraped off, and proteins were isolated as per standard protocol using standard RIPA lysis buffer. Twenty micrograms of protein were loaded on a $10 \%$ SDS gel and electrophoresed followed by Western blot analysis for PARP and caspase 8 (A). Quantitative analysis was done to determine percent apoptotic cells (B).

A

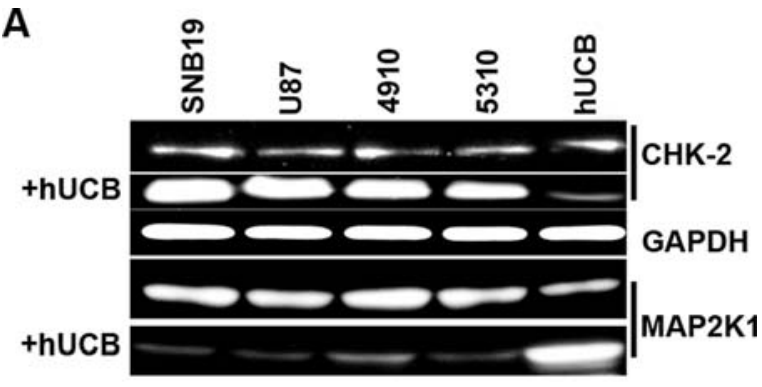

B

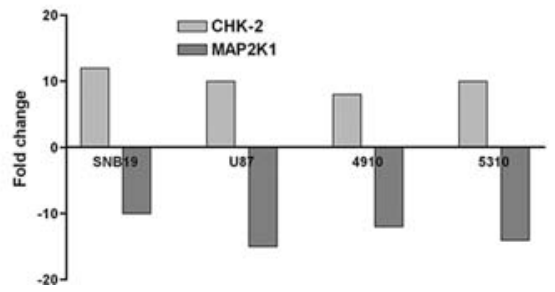

Figure 5. To determine the involvement of cell cycle regulatory molecules and the RAS signaling pathway in glioma cells in contact with hUCB stem cells, a modified Boyden's chamber was used as previously described. The chamber consisted of a porous membrane $(0.22 \mu \mathrm{m}$ pore size $)$, which was coated with Matrigel to facilitate the growth of cells on both the upper and lower surfaces. Human umbilical cord blood stem cells stained with lipophilic cell tracker red dye were grown on the upper surface and glioma cells (SNB19, U87, 5310 or 4910) were grown on the lower surface. The cells were allowed to grow for $72 \mathrm{~h}$ under standard cell culture conditions, after which the lower glioma surface layer was carefully scraped out and total RNA isolated as per standard protocol. Real-time RT-PCR analysis was carried out using primers specific for cell cycle regulatory molecules and the RAS signaling pathway (A). Agarose gels were run to validate real-time RTPCR results, which indicated changes in key cell cycle regulatory and RAS pathway molecules (B).

luciferin solution as per standard protocol. From the luminescent image analysis, we observed that control mice showed the establishment of intracranial tumors at 4 weeks after implantation. In the hUCB-implanted mice, intracranial tumors failed to develop (Fig. 6A). Analysis of the H\&E stained brain sections confirmed the luminescent studies; control mice had well-established intracranial tumors while mice treated with hUCB stem cells had minimal tumor establishment (Fig. 6A). Quantitative analysis of tumor volume by measurement of luminescence showed that control mice had the largest tumor volume with $(4.75 \pm 0.6) \times 10^{6}$ photons $/ \mathrm{sec}$ whereas nude mice implanted with both U87 and hUCB stem cells showed minimal luciferase activity of $(1 \pm 0.5) \times 10^{6}$ photons/sec (Fig. 6B).

\section{Discussion}

Stem cells are capable of self-renewal and have the ability to differentiate into a variety of tissues. For these reasons, stem cells hold great promise for practical clinical applications in 'regenerative medicine' or 'tissue engineering'. These defining characteristics and the number of particular human stem cells vary depending on the developmental stage of organism, its age, and health status. While cord blood (CB) hematopoietic stem cells are well characterized and have been clinically applied in transplantation for almost 20 years, the existence of $\mathrm{CB}$ non-haematopoietic stem cells has been recognized fairly recently (19-22), and their frequency in cord blood is still in dispute.

Umbilical cord blood (UCB) is a rich source of haematopoietic stem/progenitor cells, which is useful for clinical applications $(23,24)$. The data concerning the presence of MSC in UCB are controversial. Recent studies have shown that cord vasculature contains a high number of MSC-like elements that form colonies of fibroblastoid cells, which may be successfully expanded in culture. These MSC-like cells contain no endothelium- or leukocyte-specific antigens but express $\alpha$-smooth muscle actin and several mesenchymal cell markers. Therefore, umbilical cord/placenta stroma could be regarded as an alternative source of MSC for experimental and clinical needs (22). 


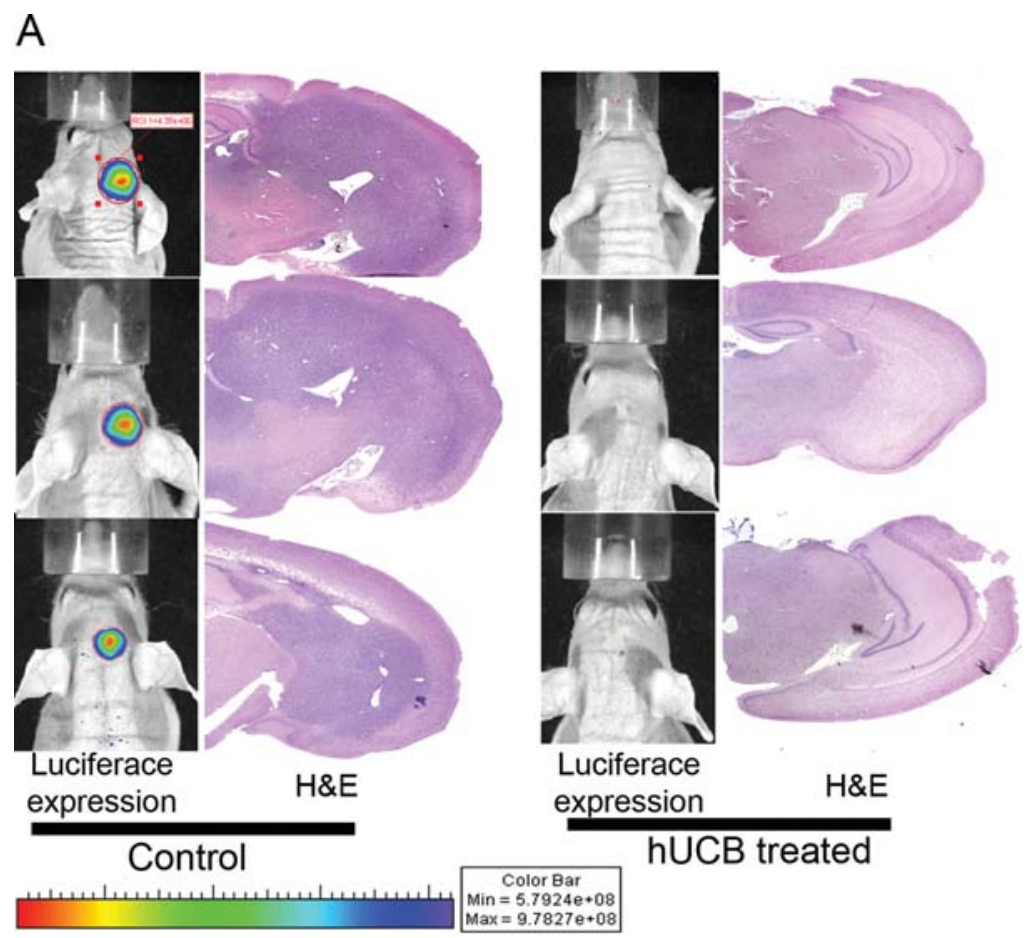

B

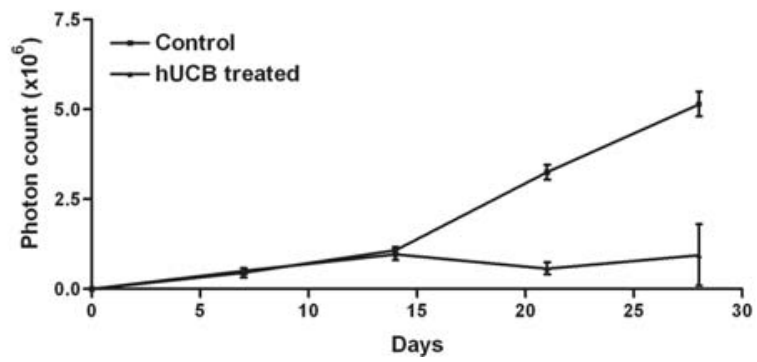

Figure 6. To determine the anti-tumor effect of hUCB stem cells in in vivo conditions, we implanted nude mice with $1 \times 10^{6}$ luciferase-expressing U87 cells. Seven days after implantation, mice were implanted again with $1 \times 10^{5}$ hUCB cells or $10 \mu 1$ of PBS at the site of U87 implantation. Tumor growth was monitored by periodic imaging with the Xenogen imaging system after intraperitoneal injections of luciferin solution as per standard protocols. Four weeks after treatment, mice were sacrificed and brains harvested followed by paraffin embedding and sectioning. The paraffin sections were H\&E stained for visualization of tumor cells (A). Tumor burden was quantified as photon count and is graphically represented (B).

To isolate cord blood stem cells, we targeted cells enriched in CD44 and CD133. Initially, we used the RosetteSep ${ }^{\circledR}$ antibody cocktail, which crosslinks unwanted cells in human cord blood to multiple red blood cells (RBC) and forms immunorosettes. This cocktail consists of antibodies that are bound in bispecific TAC which are directed against cell surface antigens on human hematopoietic cells (CD3, CD14, CD19, CD38 and CD66b) and glycophorin A on red blood cells. This enrichment leaves the MSC untouched and enabled us to isolate CD44 and CD133 positive cells. CD133+ cells are considered to be a population of non-committed early progenitors capable of self-renewing and differentiating into blood cells and other cell types. Thus, CD133 seems to be a marker associated with more primitive stem cell phenotype than CD34 (25). CD44 adhesion molecules represent a large family of transmembrane glycoproteins. Alternatively spliced additional exons may be inserted into the extracellular domain of CD44, thereby determining the structure and differential function of the multiple CD44 splice variants (26). Further, complexity of this family of adhesion molecules is determined by the fact that the extracellular domain of CD44 also contains $\mathrm{N}$ - and O-linked glycosylation sites, yielding various glycoforms of CD44. Importantly, the ligand-binding affinity of CD44 depends on its glycosylation pattern (27). Previous studies performed in Sackstein's laboratory demonstrate that a sialofucosylated glycoform of CD44 expressed on human hematopoietic cells, which was called hematopoietic cell E-/Lselectin ligand (HCELL), was a potent E-selectin ligand (28). Thus, the selection of CD44 and CD133 positive cells would provide a level of primitiveness and enable the cells to possess adhesion characteristics.

One of the hallmarks of stem cell characters is the ability to form embryoid bodies in methyl cellulose-containing media. In our study, we show that the CD44 and CD133 positive umbilical cord blood cells possess the ability to form embryoid bodies, thereby validating the stem cell property of these isolated cells.

The use of the modified Boyden's chamber provides a unique way to show the interaction of cells while preventing cross contamination of cells that are important for protein and 
RNA analysis. Thus far, we have been unable to find reports that utilize this method to determine cellular interactions. Previous reports have shown the cytotoxicity of human umbilical cord blood-derived mesenchymal stem cells against human malignant glioma cells (16) and suggest that hUCBderived MSC, with or without activation, have significant cytotoxicity against human malignant glioma cells. These researchers used the co-culture method to determine the interaction of hUCB stem cells with glioma cells. In our study, we have modified the co-culture method, thereby enabling us to separate the cells while at the same time not inhibiting cellular contact. In the present study, we have attempted to show that cell contact between hUCB stem cells and glioma cells is required for the initiation of cytotoxicity on the glioma cells. From our TUNEL assay studies, we observed that DNA damage occurred in all conditions where glioma cells were cultured with hUCB cells, whereas no damage was observed in control conditions. These results indicate that cell contact is required for initiation of DNA fragmentation. When glioma cells were cultured with hUCB-conditioned media, no DNA damage in glioma cells was observed (data not shown). These results demonstrate the involvement of certain cell surface molecules present on hUCB stem cells, which may be necessary for induction of cytotoxicity. From our membrane dye transfer studies, we observed that hUCB cells dyed with lipophilic dye caused a transfer of the lipophilic dye to the glioma cells; these results validate the involvement of cell surface components as initiators of cell-to-cell contact. No significant differences were observed between the glioma cell lines U87 and SNB19 and the glioma xenografts 4910 and 5310 .

Adapter molecules function as the cellular scaffolding upon which signal transduction complexes are assembled. Many adapter molecules lack intrinsic effecter function, but contain one or more distinct functional domains that mediate protein-protein or protein-lipid interactions. Among the modular domains important for this function are Src homology 2 (SH2) (29) and phosphotyrosine binding (PTB) domains (30) that mediate interactions with phosphorylated tyrosine residues on other proteins, Src homology 3 (SH3) domains that associate with proline-rich domains (29), and WW (31) and PDZ (32) domains that mediate other proteinprotein interactions. Additionally, some adapters mediate protein-lipid interactions through pleckstrin homology $(\mathrm{PH})$ domains (33).

CD133 (prominin-1) was the first in a class of novel pentaspan membrane proteins to be identified in both humans and mice and was originally classified as a marker of primitive haematopoietic and neural stem cells. Due to the highly restricted expression of the CD133 family of molecules on plasma membrane protrusions and its association with membrane cholesterol, a role in the organization of plasma membrane topology has recently been assigned to this family. Studies have now confirmed the utility of CD133 as a marker of haematopoietic stem cells (34). Little is known about the ligands of CD133, but its importance as a stem cell marker is valid. The ability of CD133 to produce plasma membrane protrusions is very significant and may be responsible for initiating the cell contact via the $0.22 \mu \mathrm{m}$ porous membrane that we used in the present study. CD133 is also known to be associated with lipid rafts (35) and this may be the reason why the lipophilic dye transferred to the glioma cell layer. Interestingly, CD44 and its association with lipid rafts is also evident (36). Our FACS analysis studies conclusively demonstrate that there is transfer of cell membrane components from the hUCB stem cells to the glioma cells.

The induction of cytotoxic effect on glioma cells by the hUCB stem cells is evident from the Western blot analysis where we observed the cleavage of caspase 8 in the glioma cell layer, thereby confirming the TUNEL assay studies, and the cleavage of PARP, which indicates the initiation of DNA damage. The induction of DNA damage is indicative of apoptosis and since caspase 8 is activated, the most likely mode of apoptotic induction is the extrinsic pathway (37).

From our RT-PCR studies, we observed that the levels of CHK-2 increased up to 10 -fold in glioma cells in contact with hUCB stem cells. The checkpoint kinases CHK-1 and Cds1 (CHK-2) were first identified in fission yeast as essential for cell cycle arrest before mitosis in response to DNA damage or DNA replication blockage, respectively $(38,39)$. The increase in levels of $\mathrm{CHK}-2$ confirms the initiation of cell cycle arrest by hUCB stem cells on glioma cells. We also observed that the levels of MAP2K1 were significantly reduced (more than 15-fold) in glioma cells in contact with hUCB stem cells, thereby indicating that the MEK/RAS signaling pathway was interrupted and caused cellular shutdown, which is indicative of apoptosis. These in vitro results conclusively suggest that cellular contact is required for apoptotic induction by hUCB stem cells in contact with glioma cells.

From our in vivo studies, we observed that nude mice implanted with U87 glioma cells formed intracranial tumors. However, when followed by implantation of hUCB stem cells, intracranial tumors failed to develop, thereby indicating that the hUCB stem cells caused induction of apoptosis in glioma cells. We confirmed previous reports that hUCB stem cells are cytotoxic towards glioma cells both in vitro and in vivo and, for the first time, we have demonstrated that this cytotoxicity is effective only when hUCB stem cells are in physical contact with the glioma cells.

\section{Acknowledgements}

We thank Shellee Abraham for assistance in the manuscript preparation and Diana Meister and Sushma Jasti for the review. This study was supported by National Institute of Neurological Disorders and Stroke NS057529. The contents are solely the responsibility of the authors and do not necessarily represent the official views of NIH.

\section{References}

1. Barry FP: Biology and clinical applications of mesenchymal stem cells. Birth Defects Res C Embryo Today 69: 250-256, 2003.

2. Short B, Brouard N, Occhiodoro-Scott T, Ramakrishnan A and Simmons PJ: Mesenchymal stem cells. Arch Med Res 34: 565-571, 2003.

3. Petersen BE, Bowen WC, Patrene KD, Mars WM, Sullivan AK, Murase N, Boggs SS, Greenberger JS and Goff JP: Bone marrow as a potential source of hepatic oval cells. Science 284: 1168-1170, 1999. 
4. Sanchez-Ramos JR: Neural cells derived from adult bone marrow and umbilical cord blood. J Neurosci Res 69: 880-893, 2002.

5. Woodbury D, Reynolds K and Black IB: Adult bone marrow stromal stem cells express germline, ectodermal, endodermal, and mesodermal genes prior to neurogenesis. J Neurosci Res 69: 908-917, 2002

6. Miletic H, Fischer Y, Litwak S, Giroglou T, Waerzeggers Y, Winkeler A, Li H, Himmelreich U, Lange C, Stenzel W, Deckert M, Neumann H, Jacobs AH and von Laer D: Bystander killing of malignant glioma by bone marrow-derived tumorinfiltrating progenitor cells expressing a suicide gene. Mol Ther 15: 1373-1381, 2007.

7. Nakamura K, Ito Y, Kawano Y, Kurozumi K, Kobune M, Tsuda H, Bizen A, Honmou O, Niitsu Y and Hamada H: Antitumor effect of genetically engineered mesenchymal stem cells in a rat glioma model. Gene Ther 11: 1155-1164, 2004.

8. Nakamizo A, Marini F, Amano T, Khan A, Studeny M, Gumin J, Chen J, Hentschel S, Vecil G, Dembinski J, Andreeff M and Lang FF: Human bone marrow-derived mesenchymal stem cells in the treatment of gliomas. Cancer Res 65: 3307-3318, 2005.

9. Anderson DJ, Gage FH and Weissman IL: Can stem cells cross lineage boundaries? Nat Med 7: 393-395, 2001

10. Brazelton TR, Rossi FM, Keshet GI and Blau HM: From marrow to brain: expression of neuronal phenotypes in adult mice. Science 290: 1775-1779, 2000.

11. Kang SG, Jeun SS, Lim JY, Yoo DS, Huh PW, Cho KS, Kim DS Shin HJ, Kim JH, Kim MC and Kang JK: Cytotoxicity of rat marrow stromal cells against malignant glioma cells. Childs Nerv Syst 21: 528-538, 2005.

12. Solves P, Moraga R, Saucedo E, Perales A, Soler MA, Larrea L, Mirabet V, Planelles D, Carbonell-Uberos F, Monleon J Planells T, Guillen M, Andres A and Franco E: Comparison between two strategies for umbilical cord blood collection. Bone Marrow Transplant 31: 269-273, 2003

13. Yang SE, Ha CW, Jung M, Jin HJ, Lee M, Song H, Choi S, $\mathrm{Oh}$ W and Yang YS: Mesenchymal stem/progenitor cells developed in cultures from UC blood. Cytotherapy 6: 476-486, 2004.

14. Williams B and Allan DJ: Combination of SCF, IL-6, IL-3, and GM-CSF increases the mitotic index in short term bone marrow cultures from acute promyelocytic leukemia (APL) patients. Cancer Genet Cytogenet 91: 77-81, 1996.

15. Kang SG, Ryu CH, Jeun SS, Park CK, Shin HJ, Kim JH, Kim MC and Kang JK: Lymphokine activated killer cells from umbilical cord blood show higher antitumor effect against anaplastic astrocytoma cell line (U87) and medulloblastoma cell line (TE671) than lymphokine activated killer cells from peripheral blood. Childs Nerv Syst 20: 154-162, 2004

16. Kang SG, Jeun SS, Lim JY, Kim SM, Yang YS, Oh WI, Huh PW and Park CK: Cytotoxicity of human umbilical cord bloodderived mesenchymal stem cells against human malignant glioma cells. Childs Nerv Syst 24: 293-302, 2008

17. Ganta C, Chiyo D, Ayuzawa R, Rachakatla R, Pyle M, Andrews G, Weiss M, Tamura M and Troyer D: Rat umbilical cord stem cells completely abolish rat mammary carcinomas with no evidence of metastasis or recurrence 100 days post-tumor cell inoculation. Cancer Res 69: 1815-1820, 2009

18. Kurosawa H: Methods for inducing embryoid body formation: in vitro differentiation system of embryonic stem cells. J Biosci Bioeng 103: 389-398, 2007.
19. Buzanska L, Machaj EK, Zablocka B, Pojda Z and DomanskaJanik K: Human cord blood-derived cells attain neuronal and glial features in vitro. J Cell Sci 115: 2131-2138, 2002.

20. Erices A, Conget $\mathrm{P}$ and Minguell JJ: Mesenchymal progenitor cells in human umbilical cord blood. Br J Haematol 109: 235-242, 2000.

21. Goodwin HS, Bicknese AR, Chien SN, Bogucki BD, Quinn CO and Wall DA: Multilineage differentiation activity by cells isolated from umbilical cord blood: expression of bone, fat, and neural markers. Biol Blood Marrow Transplant 7: 581-588, 2001.

22. Romanov YA, Svintsitskaya VA and Smirnov VN: Searching for alternative sources of postnatal human mesenchymal stem cells: candidate MSC-like cells from umbilical cord. Stem Cells 21: 105-110, 2003.

23. Hows JM: Status of umbilical cord blood transplantation in the year 2001. J Clin Pathol 54: 428-434, 2001.

24. Huss R: Isolation of primary and immortalized CD34-hematopoietic and mesenchymal stem cells from various sources. Stem Cells 18: 1-9, 2000.

25. Handgretinger $R$, Gordon $P R$, Leimig $T$, Chen $X$, Buhring HJ, Niethammer D and Kuci S: Biology and plasticity of CD133+ hematopoietic stem cells. Ann N Y Acad Sci 996: 141-151, 2003.

26. Khaldoyanidi S, Moll J, Karakhanova S, Herrlich P and Ponta H: Hyaluronate-enhanced hematopoiesis: two different receptors trigger the release of interleukin-1beta and interleukin-6 from bone marrow macrophages. Blood 94: 940-949, 1999.

27. Katoh S, Zheng Z, Oritani K, Shimozato T and Kincade PW: Glycosylation of CD44 negatively regulates its recognition of hyaluronan. J Exp Med 182: 419-429, 1995.

28. Dimitroff CJ, Lee JY, Rafii S, Fuhlbrigge RC and Sackstein R: CD44 is a major E-selectin ligand on human hematopoietic progenitor cells. J Cell Biol 153: 1277-1286, 2001.

29. Schlessinger J: SH2/SH3 signaling proteins. Curr Opin Genet Dev 4: 25-30, 1994.

30. Forman-Kay JD and Pawson T: Diversity in protein recognition by PTB domains. Curr Opin Struct Biol 9: 690-695, 1999.

31. Sudol M: Structure and function of the WW domain. Prog Biophys Mol Biol 65: 113-132, 1996.

32. Bezprozvanny I and Maximov A: PDZ domains: more than just a glue. Proc Natl Acad Sci USA 98: 787-789, 2001.

33. Lemmon MA and Ferguson KM: Signal-dependent membrane targeting by pleckstrin homology $(\mathrm{PH})$ domains. Biochem $\mathrm{J} 350$ : $1-18,2000$

34. Mizrak D, Brittan M and Alison MR: CD133: molecule of the moment. J Pathol 214: 3-9, 2008.

35. Giebel B, Corbeil D, Beckmann J, Hohn J, Freund D, Giesen K, Fischer J, Kogler G and Wernet P: Segregation of lipid raft markers including CD133 in polarized human hematopoietic stem and progenitor cells. Blood 104: 2332-2338, 2004.

36. Stern R: Hyaluronan catabolism: a new metabolic pathway. Eur J Cell Biol 83: 317-325, 2004.

37. Harada $\mathrm{H}$ and Grant S: Apoptosis regulators. Rev Clin Exp Hematol 7: 117-138, 2003.

38. Murakami $\mathrm{H}$ and Okayama $\mathrm{H}$ : A kinase from fission yeast responsible for blocking mitosis in $\mathrm{S}$ phase. Nature 374: 817-819, 1995.

39. Walworth N, Davey S and Beach D: Fission yeast chk1 protein kinase links the rad checkpoint pathway to cdc2. Nature 363: 368-371, 1993. 